\title{
Compact Low Weight High Gain Broadband Antenna by Polarization-Rotation Technique for X-Band Radar
}

\author{
Amir Reza Dastkhosh, ${ }^{1}$ Hamidreza Dalili Oskouei, ${ }^{2}$ and Gholamreza Khademevatan ${ }^{2}$ \\ ${ }^{1}$ Sahand University of Technology, P.O. Box 51335-1996, Tabriz, Iran \\ ${ }^{2}$ Shahid Sattari Aeronautical University of Science \& Technology, P.O. Box 13846-63113, Tehran, Iran
}

Correspondence should be addressed to Amir Reza Dastkhosh; amir_reza_dastkhosh@yahoo.com

Received 8 October 2013; Revised 13 January 2014; Accepted 24 January 2014; Published 28 April 2014

Academic Editor: Felipe Cátedra

Copyright (C) 2014 Amir Reza Dastkhosh et al. This is an open access article distributed under the Creative Commons Attribution License, which permits unrestricted use, distribution, and reproduction in any medium, provided the original work is properly cited.

Less efficiency and gain is achieved by existence of aperture blocking phenomena in cassegrain antenna caused by the presence of subreflector or antenna feed. Also, length of feed cables causes delay which is another undesirable problem in antennas, since errors and less precision of detecting targets are created. To overcome these problems, low weight and compact optimized polarizationrotation monopulse cassegrain antenna is designed in this paper. The goal of our proposed rotating antenna is achievement of sum and difference patterns for target tracking in monopulse radar. In our work, left part of hyperbolic subreflector instead of right one has been used for reducing size of the antenna. The antenna is fabricated by grid wires instead of solid sheet metal reflectors and with composite technology for decreasing its weight. Width and volume of the antenna reduce by about $50 \%$ in comparison to other reflector antennas. This antenna has been simulated and manufactured in X-band and simulation data are in good agreement with measured ones. The antenna has the average gain of $35 \mathrm{~dB}$ from $8.5 \mathrm{up}$ to $9.5 \mathrm{GHz}$. Also the antenna feed bandwidth is more than $50 \%$ and the antenna has efficiency of about $50 \%$ from 8 up to $10 \mathrm{GHz}$.

\section{Introduction}

Antennas are mostly usable in wireless communications and military applications. In military applications, radars are the most applicable parts. In military antennas we can mention microstrip antennas, phased array antennas, and reflector antennas like cassegrain antennas and fractal antennas, and by techniques such as DGS (defected ground structure), EBG (electromagnetic band gap), and FSS (frequency selective surface) the antenna efficiency can be improved. Reflector antennas especially composite structure ones are more applicable. Sieur Guillaume Cassegrain was a French sculptor who invented a form of reflecting telescope. A cassegrain telescope consists of primary and secondary reflecting mirrors. This antenna is used in many fields like astrology, optical physics, and also military surveillance systems. A surveillance system is capable of establishing a protective shield roughly 100 kilometers in diameter over an airport, military installation, or a small city (Figures 1 and 2). For this surveillance radar system, its antenna can be designed in the form of two reflecting dishes and a feed, based on the principle of the cassegrain optical telescope. There are a variety of shapes and sizes available, all described by the same set of equations. The essential performance of a cassegrain double-reflector system may be easily analyzed by means of the equivalent-parabola single-reflector concept [1-6]. Meanwhile, in designing radar antennas, fewer errors in detecting radar targets and more compact and efficient antennas are main goals. Also designing optimized and/or EBG feed for reflector antennas is a way for increasing efficiency of reflector antennas [7-12]. Moreover other techniques are available for reducing the aperture blocking by the subdish of the cassegrain system [13-19]: one method minimizes the blocking by optimizing the geometry of the feed and subdish; other methods avoid the blocking by means of polarization-rotation schemes. The latter methods are available for any application not requiring 


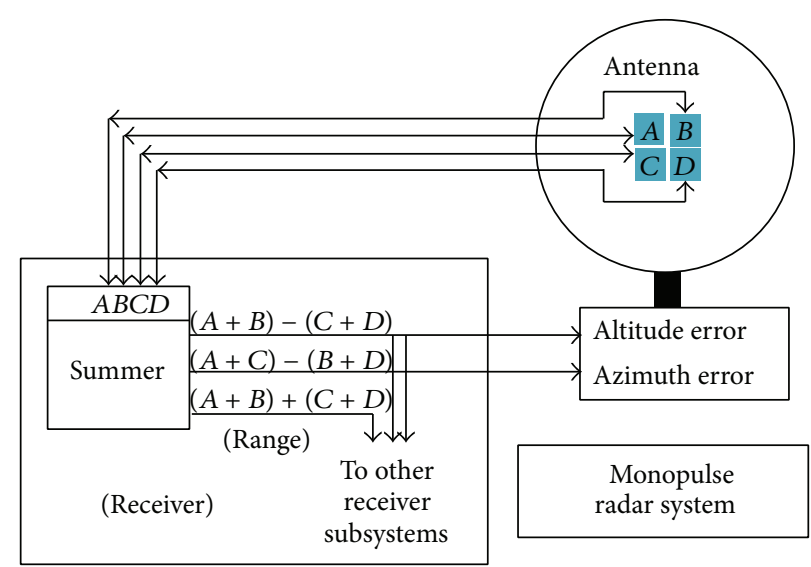

FIgURE 1: Monopulse radar system.

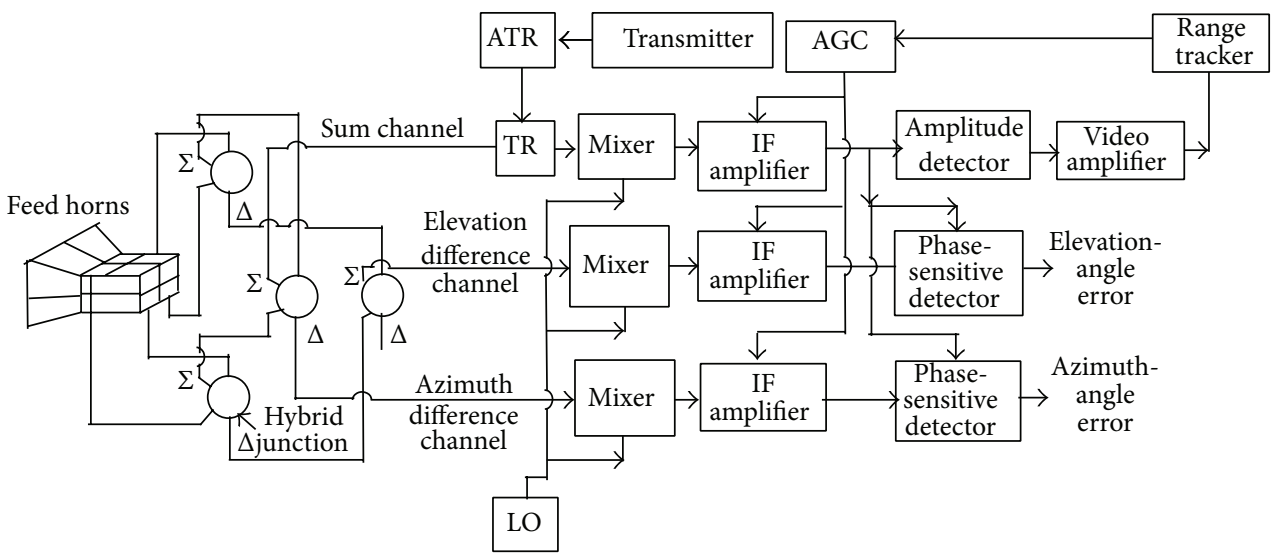

FIGURE 2: A tracking radar system.

polarization diversity, and an optimized set of polarizationoperative surfaces has been developed for these cassegrain antennas. Experimental results that have been presented for practical antennas of both types illustrate the feasibility of these principles. As we know, when the wavelength is large compared to physical dimensions, the reflector antenna can be constructed easier by grid wires instead of solid sheet metal, so the antenna weight will be reduced [20-24]. Polarization-rotation reflector that is used here consists of a grid wire spaced approximately $3 \lambda / 8$ from a ground plane or main parabolic reflector and has more than 30 percent bandwidth. In this design rule, subreflector is another grid wire in front of main reflector. The new polarization-rotation cassegrain radar antenna with grid wire main reflector and also grid wire subreflector with high gain and efficiency has been presented in this work. Designed composite structure cassegrain antenna specifications are

(1) feeding antenna in vertex of parabolic main reflector that omits the cable delays,

(2) being more compact and of low weight,
(3) having ability to transmit high power by using horn antenna feed.

Design formulas, theoretical performance, and some experimental results for this antenna are given in this work.

\section{Antenna Design}

Figure 1 shows the skyguard monopulse radar system for obtaining sum and difference patterns for detecting targets [25-29]. The monopulse radar detects targets by searching antenna and tracks them by comparing sum and difference patterns of the tracking antenna. For this system and for detecting targets beyond 200 kilometers, we must design an antenna that has the minimum gain of $34 \mathrm{~dB}$. Specifications of this antenna must be as follows.

Weight: less than $10 \mathrm{~kg}$ (because it is a rotating antenna).

Technical specifications (over the frequency band of $9 \pm$ $0.5 \mathrm{GHz})$ : 
(i) gain (one way, sum channel) $>34 \mathrm{~dB}$ (typical: $35 \mathrm{~dB}$ );

(ii) $3 \mathrm{~dB}$ beamwidth (sum channel) $=2.8 \pm 0.5^{\circ}$;

(iii) $20 \mathrm{~dB}$ beamwidth (sum channel) $=6 \pm 1^{\circ}$;

(iv) gain (difference channels) $>28 \mathrm{~dB}$;

(v) beamwidth (difference channels $-15 \mathrm{~dB}$ relative to sum average over frequency band, swept frequency) $<7.2^{\circ}$;

(vi) sidelobe level (sum channel, elevation, and azimuth) (see Table 1);

(vii) sidelobe level (difference channels relative to sum max) (see Table 2).

(viii) gain (one way, sum channel): $34 \mathrm{~dB}$ (typical: $35 \mathrm{~dB})$;

(ix) $3 \mathrm{~dB}$ beamwidth (sum channel) $=2.8^{\circ}$;

(x) $20 \mathrm{~dB}$ beamwidth (sum channel) $=7^{\circ}$;

(xi) gain (difference channels): $28 \mathrm{~dB}$;

(xii) beamwidth (difference channels $-15 \mathrm{~dB}$ relative to sum average over frequency band, swept frequency): $7^{\circ}$;

(xiii) sidelobe level (sum channel, elevation, and azimuth) (see Table 3);

(xiv) sidelobe level (difference channels relative to sum max) (see Table 4).

For designing the mentioned antenna, we begin from a common reflector antenna. The gain of reflector antenna can be calculated by

$$
G=\varepsilon_{\mathrm{ap}} \frac{4 \pi}{\lambda^{2}} A_{\mathrm{ap}}=\varepsilon_{\mathrm{ap}}\left(\frac{\pi d}{\lambda}\right)^{2},
$$

where $\varepsilon_{\mathrm{ap}}$ is the aperture efficiency and is defined by

$$
\varepsilon_{\text {ap }}=e \varepsilon_{t} \varepsilon_{1} \varepsilon_{2} \varepsilon_{3} \cdots .
$$

And $e$ is the radiation efficiency and represents the ohmic losses $(e \approx 1)$, and $\varepsilon_{t}$ is the gain loss due to an aperture illumination that is tapered relative to uniform illumination which produces the maximum gain. The remaining efficiency factors are spillover or feed efficiency, random surface error factor, and so forth [4-6]. Also in this cassegrain antenna design method, the feed antenna is near the apex of main reflector and the feed waveguide and cables' lengths are reduced; therefore it causes reduced loss and noise. In this technique, the feed antenna is placed in vertex of parabolic main reflector. Moreover, in previous works $[4,5]$ usually right part of hyperbolic subreflector is used as subreflector, but in our work for decreasing volume of the antenna we used left part of hyperbolic subreflector. The antenna is fabricated in a way that the virtual focal point of hyperbolic subreflector (fh2) and the focal point of parabolic main reflector (fpl) are the same as illustrated in Figure 3. Energy from the feed horn with vertical polarization illuminates the secondary hyperbolic subreflector parallel vertical wires which reflect the whole wave back to the parabolic main reflector and parallel parabolic wires. Also, in our design the parallel
TABLE 1

\begin{tabular}{lcc}
\hline Angular interval & Maximum value & $\begin{array}{c}\text { Average over freq. band } \\
\text { (swept frequency) }\end{array}$ \\
\hline$<10^{\circ}$ & $<-20 \mathrm{~dB}$ & $<-22 \mathrm{~dB}$ \\
$10 \sim 30^{\circ}$ & $<-24 \mathrm{~dB}$ & $<-25 \mathrm{~dB}$ \\
$>30^{\circ}$ & $<-30 \mathrm{~dB}$ & $<-34 \mathrm{~dB}$ \\
\hline
\end{tabular}

TABLE 2

\begin{tabular}{lcc}
\hline Angular interval & Maximum value & $\begin{array}{c}\text { Average over freq. band } \\
\text { (swept frequency) }\end{array}$ \\
\hline $6 \sim 10^{\circ}$ & $<-18 \mathrm{~dB}$ & $<-20 \mathrm{~dB}$ \\
$10 \sim 30^{\circ}$ & $<-20 \mathrm{~dB}$ & $<-25 \mathrm{~dB}$ \\
$>30^{\circ}$ & $<-25 \mathrm{~dB}$ & $<-28 \mathrm{~dB}$ \\
\hline
\end{tabular}

TABLE 3

\begin{tabular}{lcc}
\hline Angular interval & Maximum value & $\begin{array}{c}\text { Average over freq. band } \\
\text { (swept frequency) }\end{array}$ \\
\hline$<10^{\circ}$ & $-20 \mathrm{~dB}$ & $-21 \mathrm{~dB}$ \\
$10 \sim 30^{\circ}$ & $-25 \mathrm{~dB}$ & $-25 \mathrm{~dB}$ \\
$>30^{\circ}$ & $-31 \mathrm{~dB}$ & $-34 \mathrm{~dB}$ \\
\hline
\end{tabular}

TABLE 4

\begin{tabular}{lcc}
\hline Angular interval & Maximum value & $\begin{array}{c}\text { Average over freq. band } \\
\text { (swept frequency) }\end{array}$ \\
\hline $6 \sim 10^{\circ}$ & $-18 \mathrm{~dB}$ & $-20 \mathrm{~dB}$ \\
$10 \sim 30^{\circ}$ & $-20 \mathrm{~dB}$ & $-25 \mathrm{~dB}$ \\
$>30^{\circ}$ & $-25 \mathrm{~dB}$ & $-28 \mathrm{~dB}$ \\
\hline
\end{tabular}

parabolic wires are in front of the primary parabolic main reflector metal mesh and in position with angle of $45^{\circ}$ to the polarization of the horn feed antenna. For changing wave polarization and increasing frequency bandwidth the distance between main parabolic reflector metal mesh and parallel parabolic wires is $3 \lambda / 8$ (Figure 3 ).

When the vertically polarized wave penetrates this parabolic grid wire and main parabolic reflector metal mesh, all waves will reflect by parallel wires and parabolic main reflector. On the way back this vertically polarized wave converts to a horizontally polarized wave. Figure 4 shows how transmitted vertically polarized power of horn antenna is changed to horizontal one when it is transmitted finally. The transmitted vertically polarized signal of the horn antenna is shown in " $A$ " vector. This signal is reflected by vertical parallel hyperbolic wires in front of it and the signal reflects to parallel parabolic wires and parabolic main reflector. The " $A 1$ " component of " $A$ " vector passes thorough wires but reflects by the main parabolic reflector almost without any change because the space between parallel parabolic wires and parabolic main reflector is $3 \lambda / 8$, but " $A 2$ " component of 


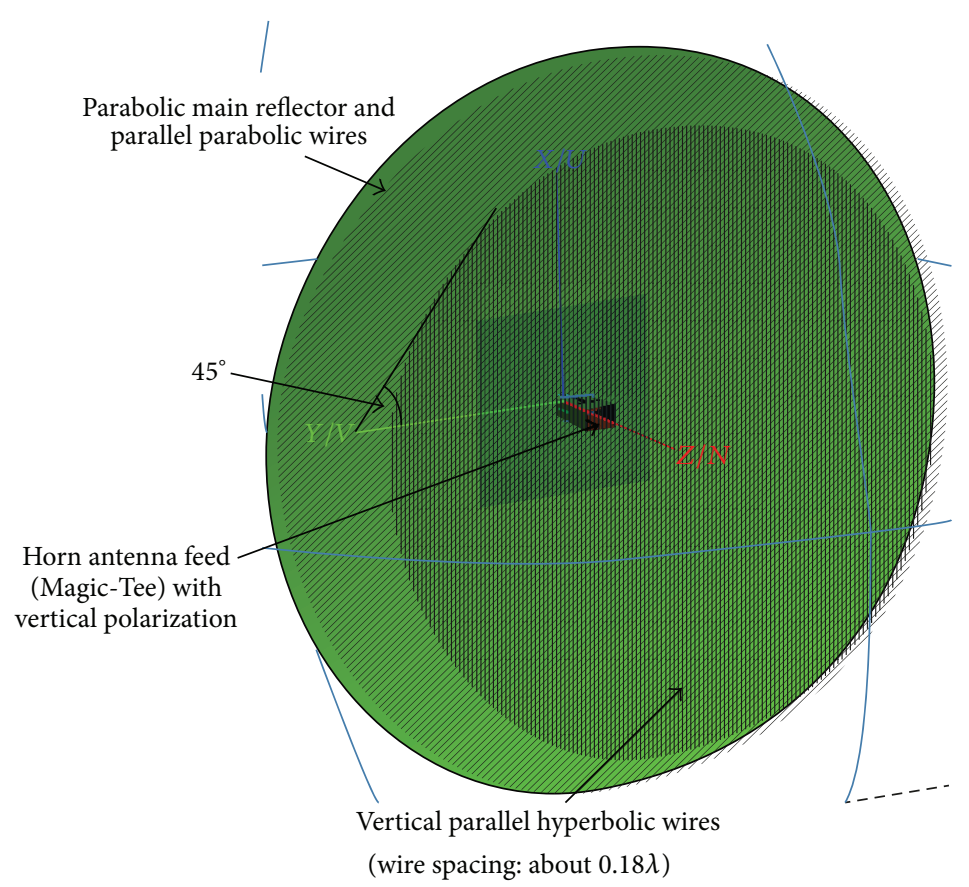

(a)

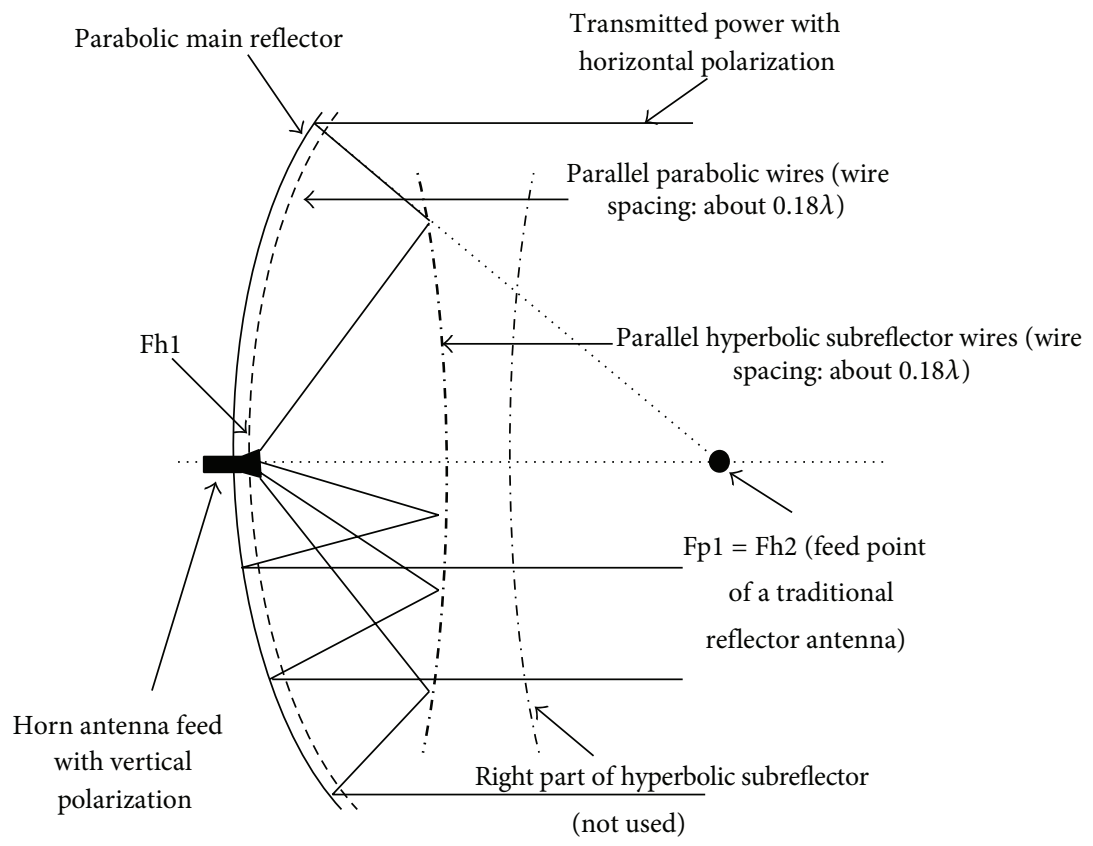

(b)

Figure 3: (a) Cassegrain antenna structure and (b) its design rule.

" $A$ " is converted to " $-A 2$ "; therefore " $B$ " vector will be the same " $A$ " vector but with $90^{\circ}$ phase shift. The operation of the antenna for received horizontally polarized signal is in the same manner to transmitted one. In manufacturing process all of the above mentioned design rules were done (Figure 5).
The space between parallel parabolic wires and parabolic main reflector is filled with composite material (honeycomb $\left(\varepsilon_{r} \approx 1.1, \tan \delta \approx 0.001\right)$ ). The dimensions related to the wires are wire diameters: about $0.0046 \lambda$; wire spacing: about $0.18 \lambda$; honeycomb thickness: $3 / 8 \lambda$. 


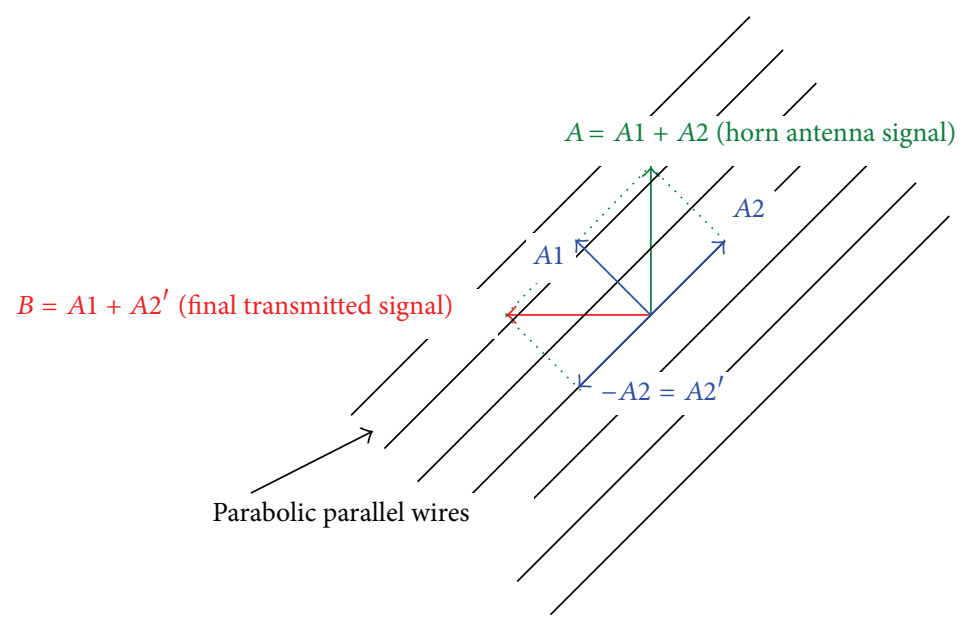

Figure 4: Polarization change of transmitted (received) signal by parabolic main reflector and wires.
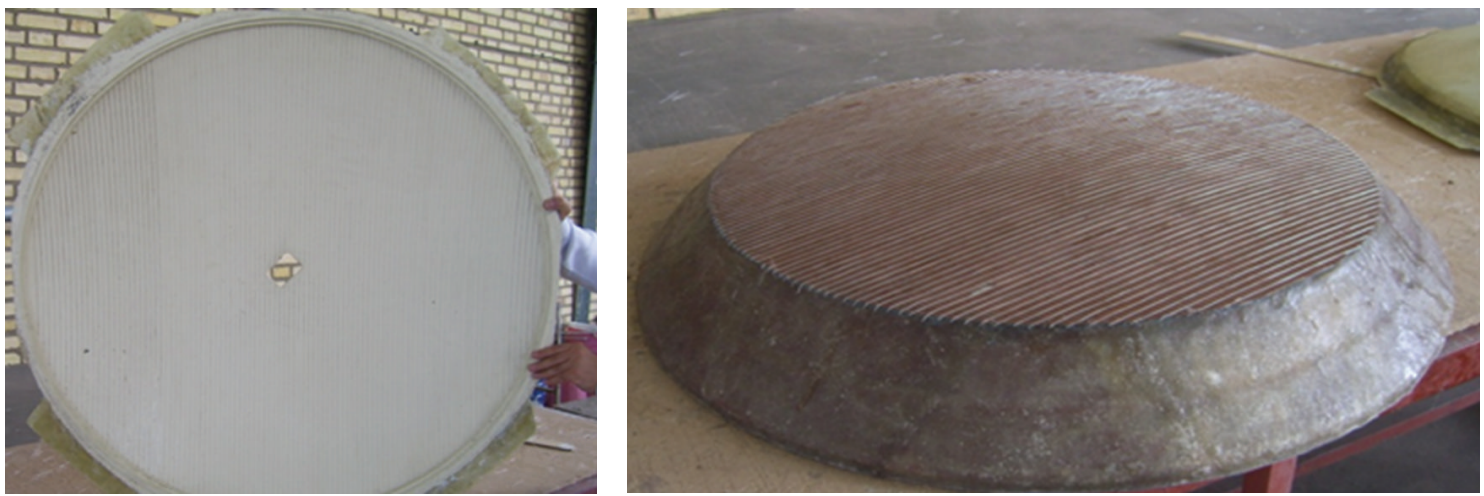

FIGURE 5: Manufacturing process.

2.1. Antenna Feed. Antenna feed consists of 4-horn array that can create 90-phase shift by using Magic-Ts. The feed and 3$D$ patterns are shown in Figures 6 and 7. The Magic-Ts create sum and difference patterns that are sum: $A+B+C+D$; difference: $(A+B)-(C+D)$ and $(A+C)-(B+D)$.

The manufactured antenna and its feed are shown in Figure 8. By using this 4-horn array antenna feed, we can obtain sum and difference antenna patterns that are used in monopulse radar [18]. The dimensions of the antenna are shown in Figure 9.

\section{Simulated and Measured Results}

This antenna was simulated with MoM/PO (method of moments/physical optics) hybridization tool, prepared by FEKO commercial software [30, 31], and fabricated and measured. For testing this antenna, we use far-field pattern formula for obtaining the region required for testing in antenna room as follows:

Diameter of antenna $=0.9 \mathrm{~m}$; frequency $=9.5 \mathrm{GHz} \Rightarrow$ $\lambda=0.0316 \mathrm{~m}$.
So we have

$$
F F=\frac{2 D^{2}}{\lambda}=\frac{1.62}{0.0316}=51.3 \mathrm{~m}
$$

So the antenna room or anechoic chamber must be so large and finally the antenna was tested in free space that transmitter and the antenna (receiver) were in the distance of 500 meters from each other. The results are shown in Figures 10, 11, and 12. Also, the return loss of the antenna was measured and simulated (Figure 13). As we can see, the antenna bandwidth is more than $50 \%$ and the antenna has efficiency of about $50 \%$ from 8 up to $10 \mathrm{GHz}$. The obtained characteristics are

\section{Weight: $6 \mathrm{~kg}$,}

Technical specifications (over the frequency band of $9 \pm$ $0.5 \mathrm{GHz}$ ):

(i) gain (one way, sum channel): $34 \mathrm{~dB}$ (typical: $35 \mathrm{~dB}$ );

(ii) $3 \mathrm{~dB}$ beamwidth (sum channel) $=2.8^{\circ}$;

(iii) $20 \mathrm{~dB}$ beamwidth (sum channel) $=7^{\circ}$; 


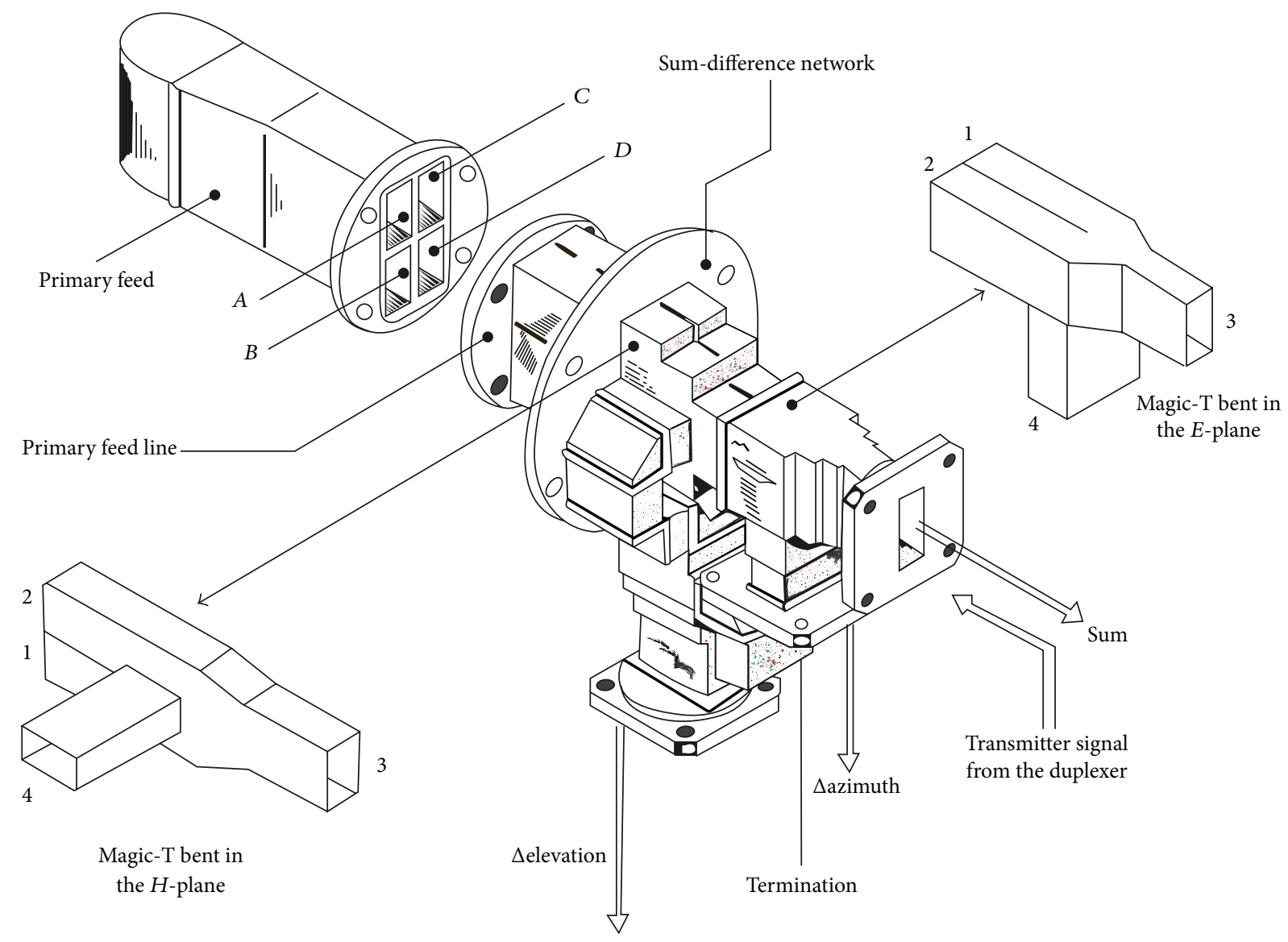

(a)

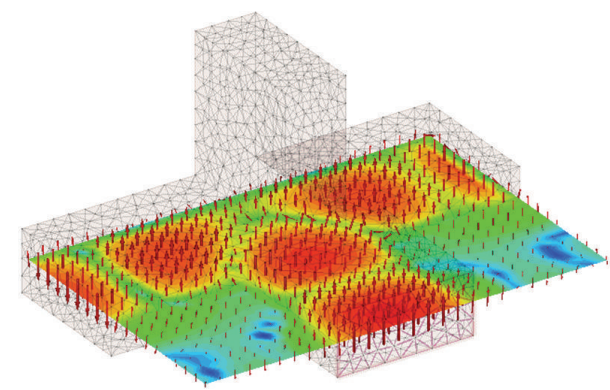

(b)

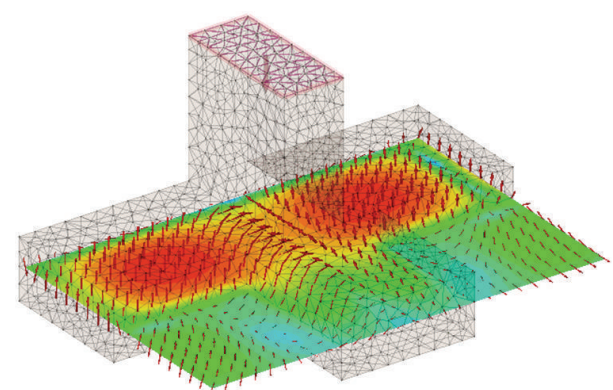

(c)

Figure 6: (a) Antenna feed. (b) E-field simulation for sum and (c) difference ports.

(iv) gain (difference channels): $28 \mathrm{~dB}$;

(v) beamwidth (difference channels $-15 \mathrm{~dB}$ relative to sum average over frequency band, swept frequency): $7^{\circ}$;

(vi) sidelobe level (sum channel, elevation, and azimuth) (see Table 3);

(vii) sidelobe level (difference channels relative to sum max) (see Table 4).

\section{Conclusion}

In this work a low-weight and compact high gain cassegrain antenna is designed and fabricated. The aperture blocking phenomena that causes decreasing of the antenna gain is omitted by optimized polarization-rotation reflector. Also by using the feed in vertex of the parabolic reflector delay lines of feed cables are omitted. For achieving low weight and compact rotating antenna, we used left part of hyperbolic as subreflector, grid wires and composite technology. By using these techniques the antenna weight is less than $6 \mathrm{Kg}$. Also, 

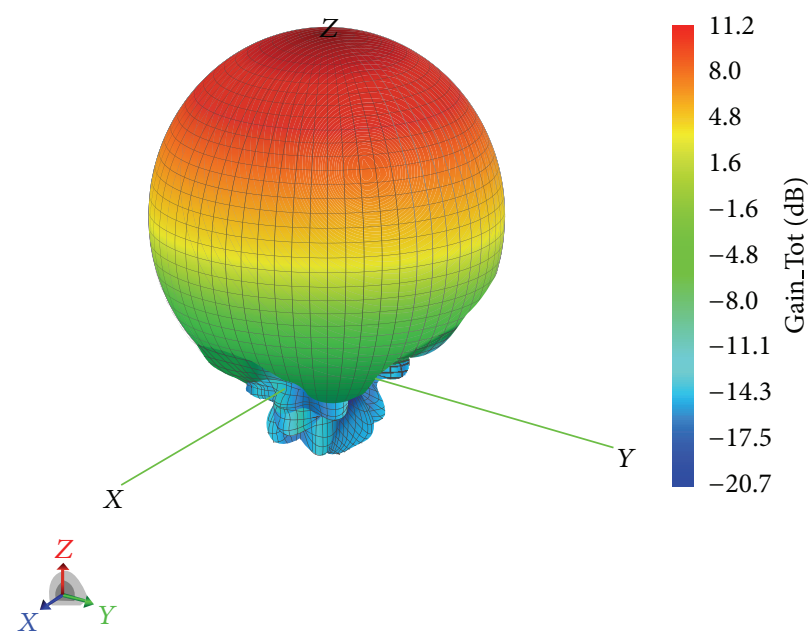

FiguRE 7: 3-D feed antenna pattern.

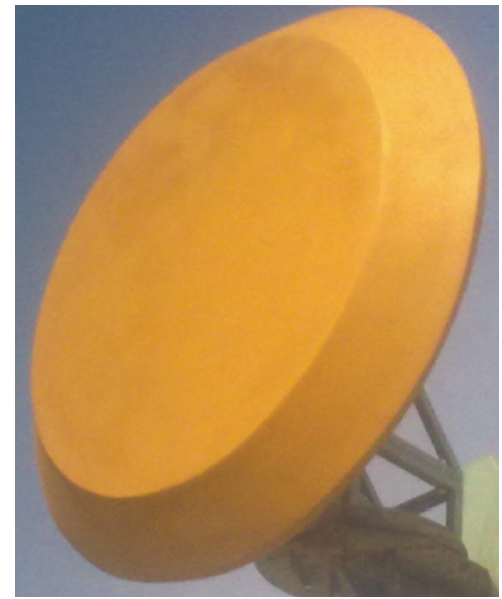

(a)

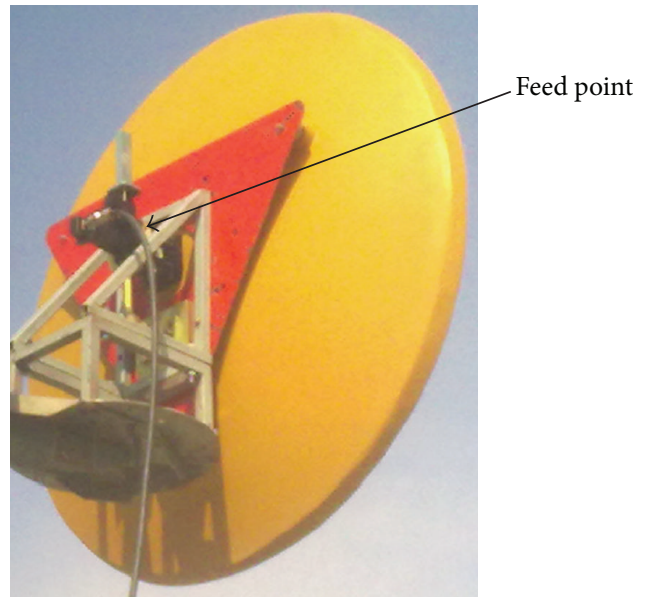

(b)

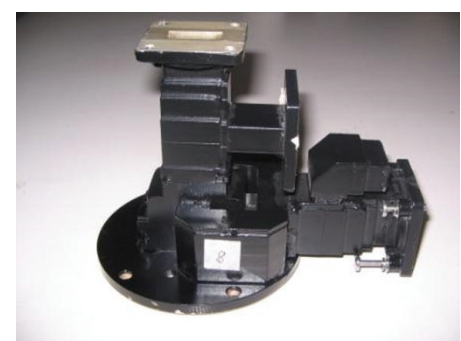

(c)

Figure 8: The manufactured cassegrain antenna under far field pattern test (free space); (a) front view; (b) back view; (c) its 4-horn array antenna feed with sum and difference port without radome (used for better efficiency).

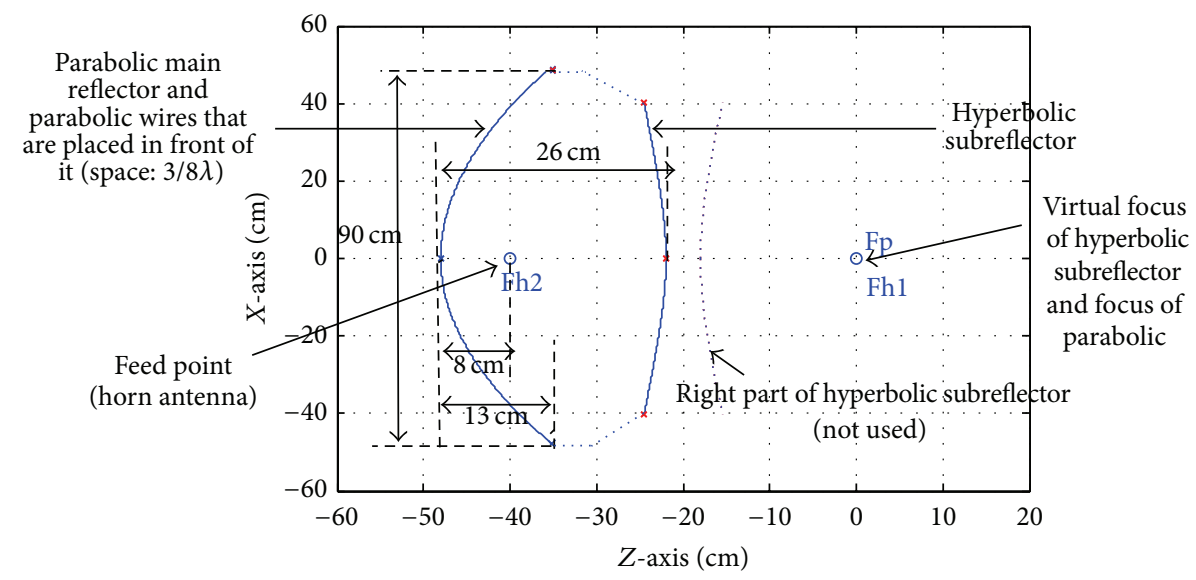

FIGURE 9: The cassegrain antenna structure and its dimensions. 


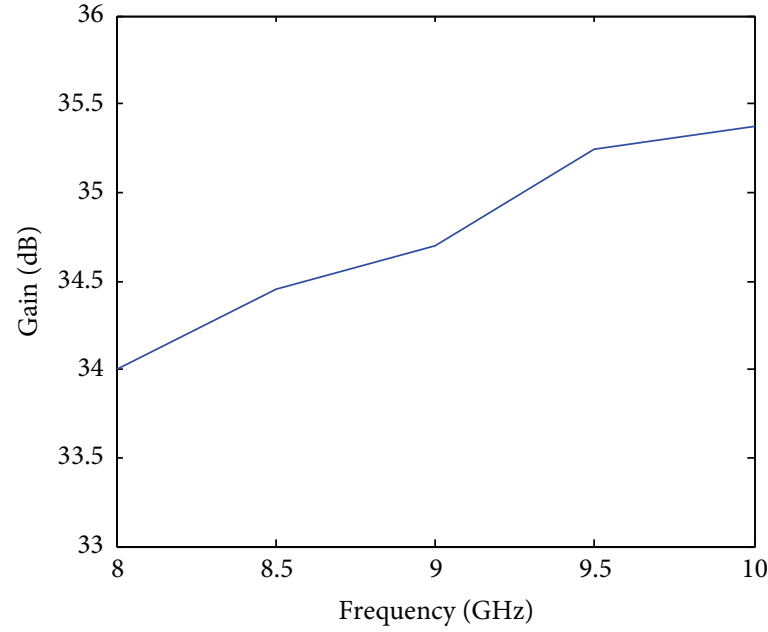

(a)

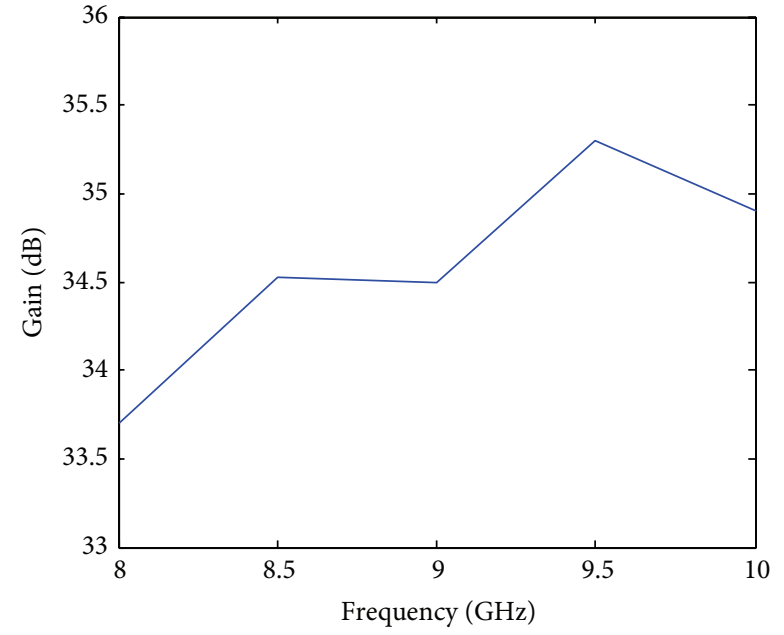

(b)

FIGURE 10: (a) Gain versus frequency of the simulated cassegrain antenna by FEKO. (b) The gain versus frequency of manufactured cassegrain antenna.

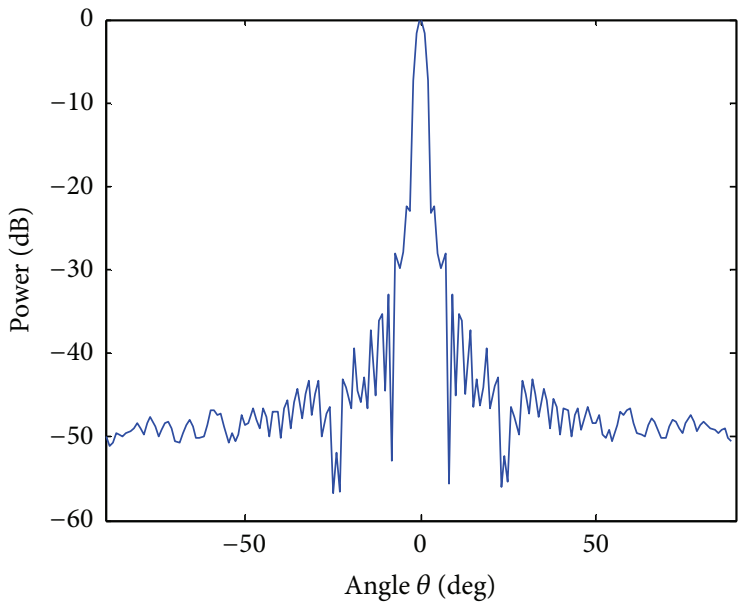

(a)

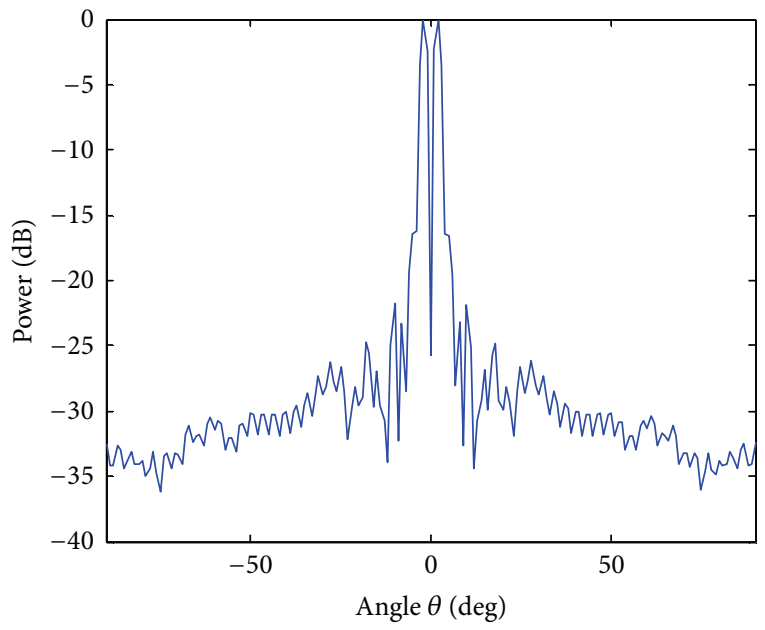

(b)

FIGURE 11: (a) The sum pattern and (b) difference pattern of simulated cassegrain antenna by FEKO at 9 GHz (normalized power).

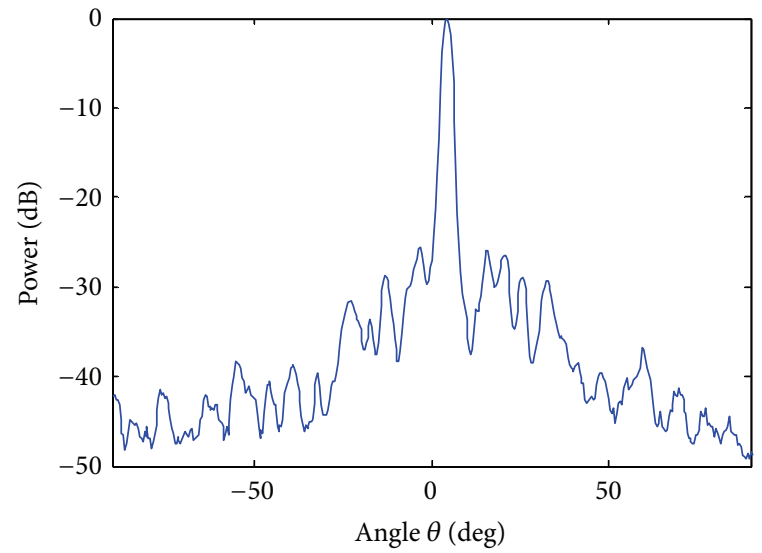

(a)

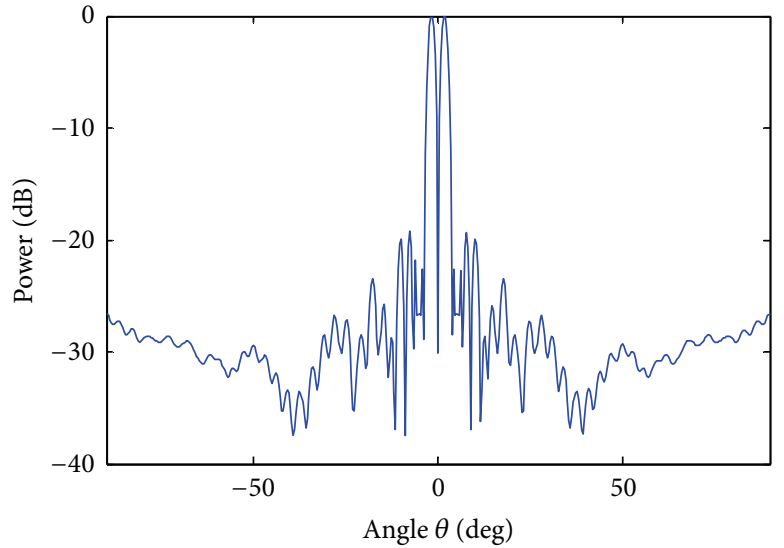

(b)

FIGURE 12: (a) The sum pattern and (b) difference pattern of measured cassegrain antenna at $9 \mathrm{GHz}$ (normalized power). 


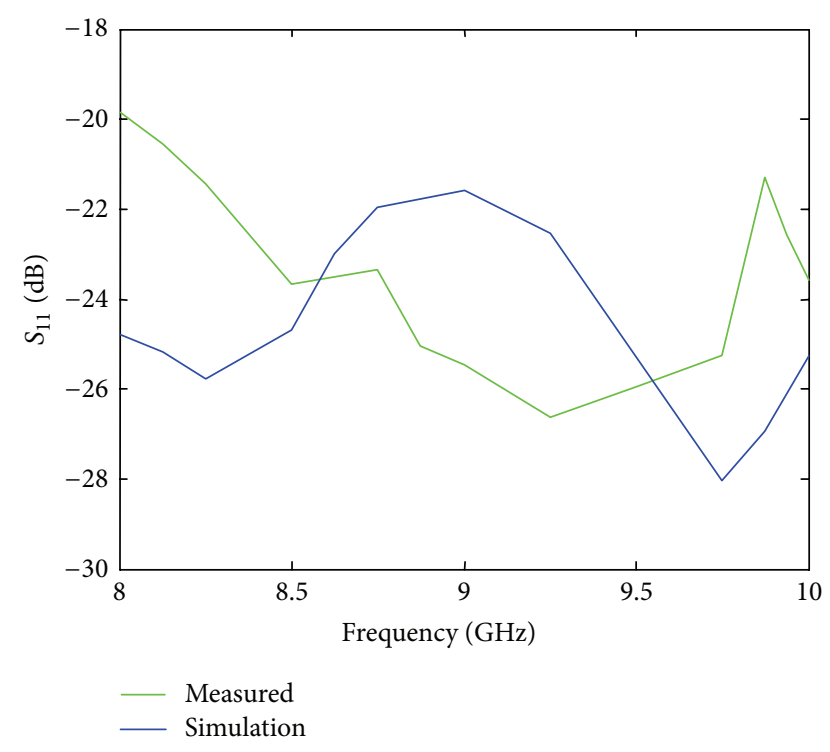

FIGURE 13: Reflection coefficient of simulated and measured antenna.

the optimized design technique was used for obtaining the best gain and size of this kind of antenna. The antenna gain is more than $34.5 \mathrm{~dB}$ from 8.5 up to $9.5 \mathrm{GHz}$. This efficient lowweight antenna is suitable for military vehicle and radars.

\section{Conflict of Interests}

The authors declare that there is no conflict of interests regarding the publication of this paper.

\section{References}

[1] L. Josefsson, "Broad-band twist reflector," IEEE Transactions on Antennas and Propagation, vol. 19, no. 4, pp. 552-554, 1971.

[2] O. A. Yurtsev, N. M. Naumovich, and E. A. Pikhta, "Numerical simulation of monopulse double-reflector antennas," in Proceedings of the 6th International Conference on Antenna Theory and Techniques (ICATT '07), pp. 229-231, IEEE, September 2007.

[3] C. Granet, "Designing classical open-cassegrain dual-reflector antennas from combinations of prescribed geometric parameters [antenna designer's notebook]," IEEE Antennas and Propagation Magazine, vol. 54, no. 2, pp. 136-147, 2012.

[4] M. I. Skolnik, Radar Handbook, McGraw-Hill Professional, 2008.

[5] M. I. Skolnik, Introduction to Radar Systems, McGraw-Hill, 2001.

[6] W. L. Stutzman and G. A. Thiele, Antenna Theory and Design, Wiley, 1988.

[7] N. Llombart, A. Neto, G. Gerini, and P. de Maagt, "Planar circularly symmetric EBG structures for reducing surface waves in printed antennas," IEEE Transactions on Antennas and Propagation, vol. 53, no. 10, pp. 3210-3218, 2005.

[8] J. M. Bell, M. F. Iskander, and J. J. Lee, "Ultrawideband hybrid EBG/ferrite ground plane for low-profile array antennas," IEEE
Transactions on Antennas and Propagation, vol. 55, no. 1, pp. 412, 2007.

[9] Y. Pang, B. Gao, and Z. Du, "Novel electromagnetic bandgap structure fabricating method," in Proceedings of the IEEE Antennas and Propagation Society International Symposium, vol. 4, pp. 372-375, IEEE, June 2002.

[10] G. Xun, T. Smyth, and W. J. Chappell, "Cofiring different dielectric constants inside LTCC for metamaterial applications," in Proceedings of the IEEE Antennas and Propagation Society International Symposium, pp. 1935-1938, IEEE, July 2006.

[11] A. Yu and X. Zhang, "A novel 2-D electromagnetic band-gap structure and its application in micro-strip antenna arrays," in Proceedings of the 3rd International Conference Microwave and Millimeter Wave Technology (ICMMT'02), IEEE, 2002.

[12] F. Yang and Y. Rahmat-Samii, Electromagnetic Band Gap Structures in Antenna Engineering, Cambridge University Press, Cambridge, UK, 2009.

[13] P. W. Harman, "Double-reflector antenna with polarizationchanging subreflector," U.S. Patent No. 3, 235, 870. 15 February 1966.

[14] B. M. Thomas, G. L. James, and K. J. Greene, "Design of wideband corrugated conical horns for Cassegrain antennas," IEEE Transactions on Antennas and Propagation, vol. 34, no. 6, pp. 750-757, 1986.

[15] S. Srikanth, "Comparison of spillover loss of offset Gregorian and Cassegrain antennas," in Proceedings of the Antennas and Propagation Society Symposium, AP-S. Digest, pp. 444-447, IEEE, June 1991.

[16] C. Menudier, R. Chantalat, E. Arnaud, M. Thevenot, T. Monediere, and P. Dumon, "EBG focal feed design optimization," IEEE Antennas and Wireless Propagation Letters, vol. 8, pp. 315-318, 2009.

[17] Y. Rahmat-Samii, "Subreflector extension for improved efficiencies in Cassegrain antennas-GTD/PO analysis," IEEE Transactions on Antennas and Propagation, vol. 34, no. 10, pp. 1266-1269, 1986.

[18] A. Tadjalli and J. Rashed-Mohassel, "Blockage minimization in Cassegrain and Gregorian reflectors with increased flexibility," in Proceedings of the 2002 IEEE Aerospace Conference, vol. 2, IEEE, 2002.

[19] D. I. L. de Villiers, "Gain ripple in small offset Gregorian antennas," in Proceedings of the IEEE International Symposium on Antennas and Propagation (APSURSI '11), pp. 2172-2175, IEEE, July 2011.

[20] C. A. Balanis, Antenna Theory: Analysis and Design, John Wiley \& Sons, 2005.

[21] P. Hannan, "Microwave antennas derived from the Cassegrain telescope," IRE Transactions on Antennas and Propagation, vol. 9, no. 2, pp. 140-153, 1961.

[22] H. Rajagopalan, A. Miura, and Y. Rahmat-Samii, "Equivalent strip width for cylindrical wire for mesh reflector antennas: experiments, waveguide, and plane-wave simulations," IEEE Transactions on Antennas and Propagation, vol. 54, no. 10, pp. 2845-2853, 2006.

[23] M. I. Astrakhan, "Reflecting and screening properties of plane wire grids," Radio Engineering, vol. 23, no. 1, p. 76, 1968.

[24] A. Miura and Y. Rahmat-Samii, "Analysis of mesh reflector antennas with complex mesh surfaces using physical optics combined with periodic method of moments," in Proceedings of the Asia-Pacific Microwave Conference (APMC '05), vol. 3, IEEE, December 2005. 
[25] S. Qian, X. Li, and B. Wang, "Ka band cassegrain monopulse antenna fed by tapered rod antennas," in Proceedings of the 8th International Symposium on Antennas, Propagation and EM Theory (ISAPE '08), pp. 39-41, IEEE, November 2008.

[26] H. Bayer, A. Krauss, R. Stephan, and M. A. Hein, "Multimode monopulse tracking feed with dual-band potential for landmobile satellite communications in Ka-band," in Proceedings of the 6th European Conference on Antennas and Propagation (EUCAP '12), IEEE, 2012.

[27] H. Meng and W. Dou, "Design of diffractive Cassegrain antenna at W band," in Proceedings of the IEEE CIE International Conference on Radar (RADAR '11), vol. 2, pp. 1176-1178, IEEE, October 2011.

[28] J. A. Martinez-Lorenzo, A. Garcia-Pino, B. Gonzalez-Valdes, and C. M. Rappaport, "Zooming and scanning Gregorian confocal dual reflector antennas," IEEE Transactions on Antennas and Propagation, vol. 56, no. 9, pp. 2910-2919, 2008.

[29] P. Mikulich, L. Yorinks, C. Profera et al., "High gain cassegrain monopulse antenna," in Proceedings of the 1968 Antennas and Propagation Society International Symposium, vol. 6, IEEE, 1968.

[30] R. F. Harrington, Time-Harmonic Electromagnetic Fields, McGraw-Hill, New York, NY, USA, 1961.

[31] https://www.feko.info/product-detail/numerical_methods/po. 

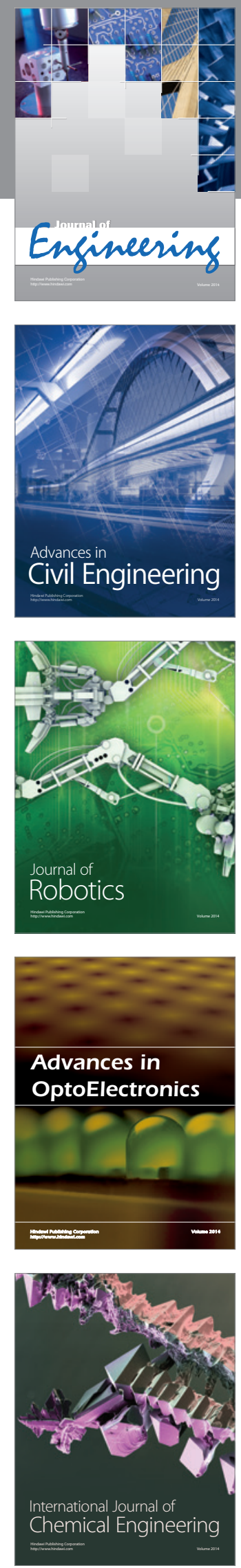

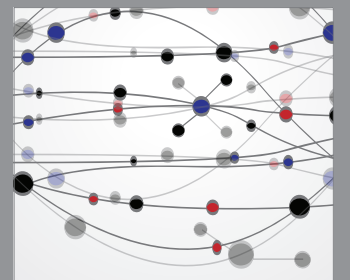

The Scientific World Journal
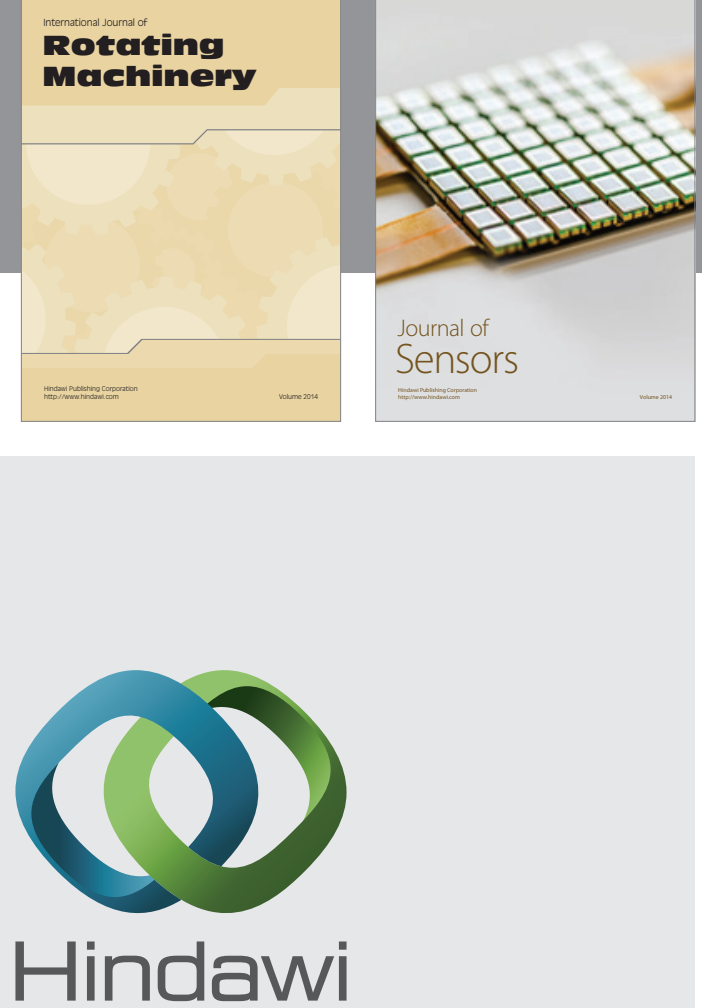

Submit your manuscripts at http://www.hindawi.com
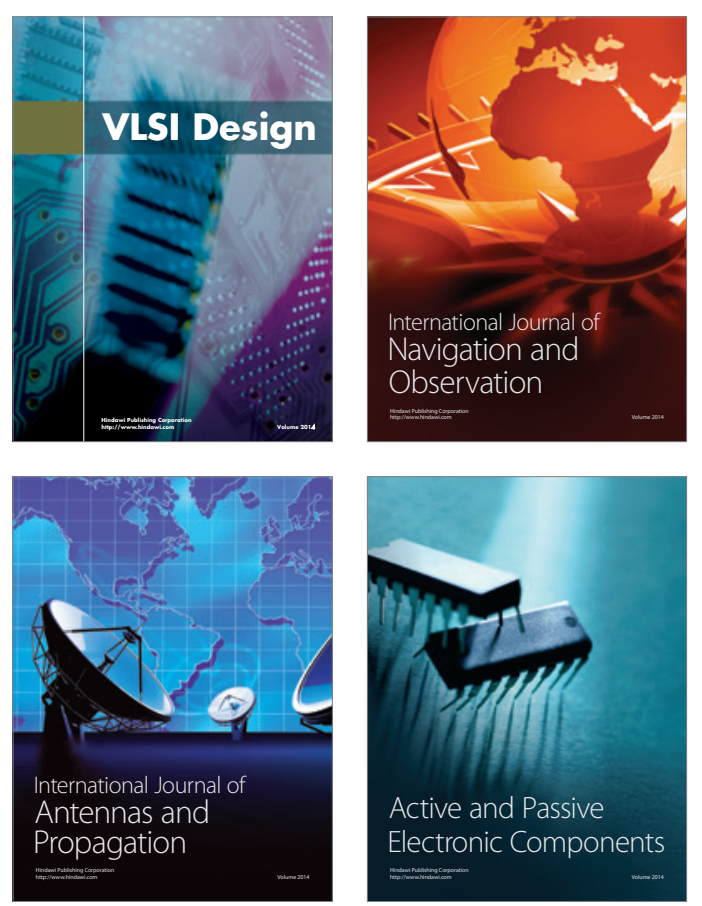
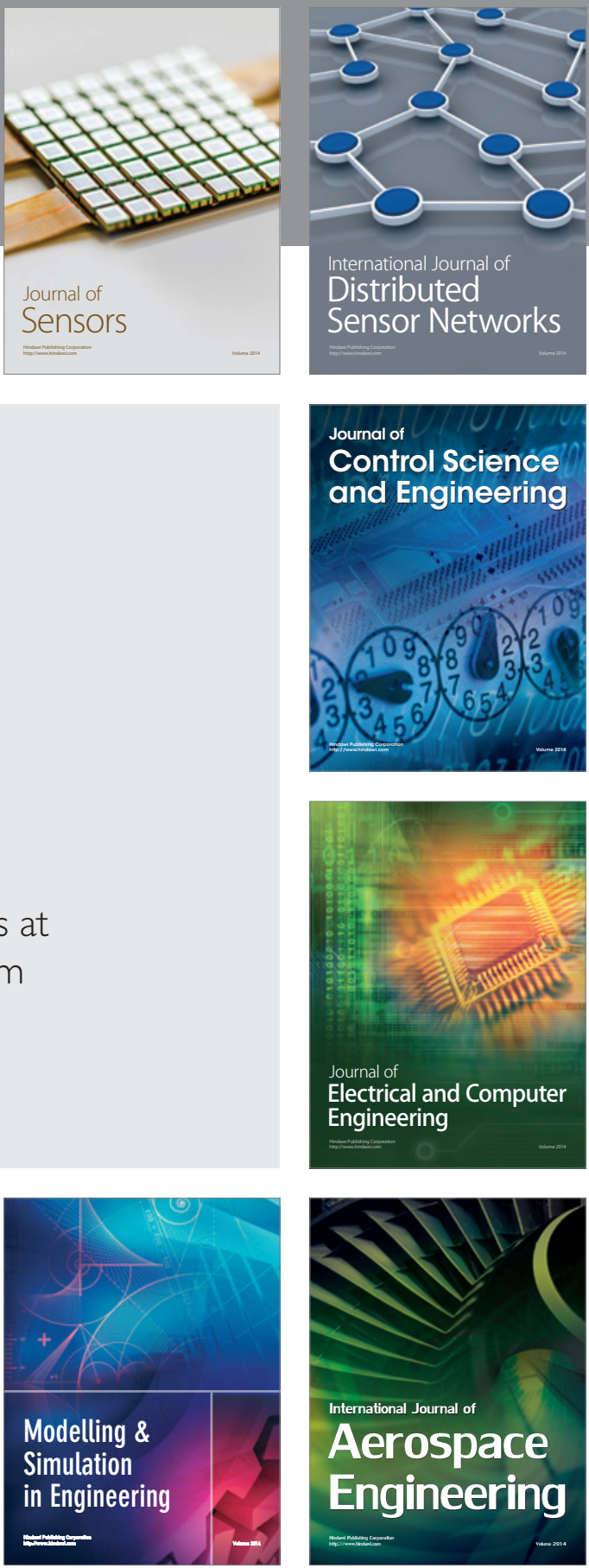

Journal of

Control Science

and Engineering
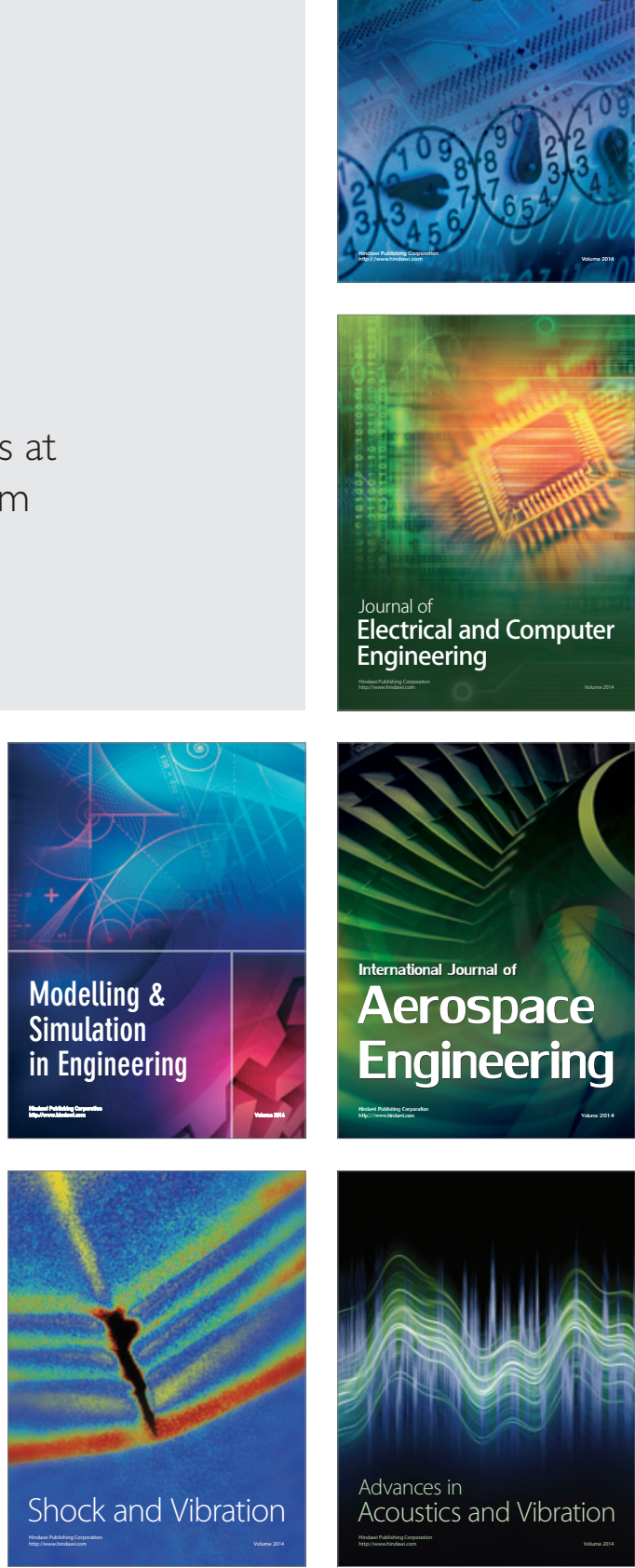